\title{
Nonimmune hydrops fetalis in a children's hospital: a six-year series
}

Zhi Min $\underline{N g}^{1}$, MBBs, MrCPCH, Meei Jiun Seet ${ }^{1}$, BM, May Na Erng ${ }^{1}$, MBBS, MrCPCH, Flynn Buendia ${ }^{1}$, MD, Alvin Shang Ming Chang $^{1}$, MBBS, FRCPE, Bhavani Sriram ${ }^{1}$, MBBS, MRCP

INTRODUCTION We aimed to study the profile of nonimmune hydrops fetalis (NIHF) in the local population and identify its outcomes and causes.

METHODS We carried out a retrospective review of the medical records in KK Women's and Children's hospital, a single tertiary referral centre, for pregnancies with an antenatal diagnosis of NIHF in the six-year period from 1 January 2005 to 31 December 2010.

RESULTS A total of 29 cases of NIHF were identified; 19 (66\%) cases underwent karyotype evaluation, 17 (59\%) underwent intrauterine infection screening, and all underwent antenatal thalassaemia screening. The median gestational age at diagnosis was 27 (range 12-37) weeks, median gestational age at birth was 33 (range 27-37) weeks, and median birth weight of live births was 2,480 (range 1,230-3,900) g. The aetiologies for NIHF were identified in $20(69 \%)$ cases, which included cardiac anomalies $(n=5)$, haematological problems $(n=4)$, congenital tumours $(n=4)$, genetic/metabolic disorders $(n=4)$ and cystic hygromas $(n=3)$. The cause of NIHF was not identified in the remaining $9(31 \%)$ cases. There were 19 live births - $8(42 \%)$ survived and $11(58 \%)$ died in the neonatal period - and one stillbirth. Nine women opted for medical termination of pregnancy following the diagnosis of NIHF.

CONCLUSION It is important to thoroughly investigate all cases of NIHF and identify its causes in order to provide appropriate antenatal and postnatal counselling. In our series, almost one-third of NIHF cases had no identified aetiology. The neonatal mortality rate was approximately $58 \%$.

Keywords: aetiology, nonimmune hydrops fetalis, outcome

\section{INTRODUCTION}

Hydrops fetalis is defined as excessive fluid accumulation within at least two fetal extravascular compartments or body cavities. It is characterised by generalised skin thickening of more than $5 \mathrm{~mm}$, placental enlargement, pericardial or pleural effusion and/or ascites.(1) With advances in prenatal diagnosis and routine immunisation of Rhesus-negative mothers, nonimmune hydrops fetalis (NIHF) is now a more frequent diagnosis, comprising $76 \%-87 \%$ of all described cases of hydrops fetalis. ${ }^{(2)}$ However, despite improvements in the diagnosis and management of NIHF, morbidity and mortality still remain significant. We aimed to study the profile of NIHF in the local population and identify its causes and outcomes in order to promote appropriate management of cases diagnosed with NIHF at our hospital.

\section{METHODS}

A retrospective review of the medical records of pregnancies with an antenatal diagnosis of NIHF during the period between 1 January 2005 and 31 December 2010 was carried out. Only cases that fulfilled the aforementioned criteria for hydrops fetalis were included in the study. These were identified by searching the birth defect database maintained by the Department of Neonatology at KK Women's and Children's Hospital $(\mathrm{KKH})$, Singapore. All cases of birth defects managed at $\mathrm{KKH}$, both antenatally and postnatally, were recorded in the database. Cases diagnosed with immune hydrops fetalis were excluded from the study.

All case notes and electronic medical records of identified cases were reviewed by the principal investigator and coinvestigators. Clinical data was collected using a coded data collection sheet. Information on maternal history, antenatal history, birth history, investigations performed, diagnosis (if known) and outcome was then transferred into a passwordlocked Microsoft Excel (Microsoft Corporation, Redmond, WA, USA) spreadsheet. The research was approved by the SingHealth Institutional Review Board based on the deidentified data set provided. Data analysis was performed only within the de-identified database.

\section{RESULTS}

We identified 29 cases with an antenatal diagnosis of NIHF in the database. The maternal demographic characteristics and key antenatal investigations are presented in Table I. The mean maternal age was 30 (range 23-42) years. None of the mothers had a history of hydrops fetalis or any significant social history. Antenatal screenings for hepatitis B, syphilis and human immunodeficiency virus were negative. Three women had pregnancy-induced hypertension, two had gestational diabetes mellitus and one had thyroid disease. One woman had a known thalassaemia trait and one woman had thalassaemia haemoglobin- $\mathrm{H}(\mathrm{HbH})$ disease. Only one of the 29 cases

'Department of Neonatology, KK Women's and Children's Hospital, Singapore

Correspondence: Dr Ng Zhi Min, Registrar, KK Women's and Children's Hospital, 100 Bukit Timah Road, Singapore 229899. ng.zhi.min@kkh.com.sg 
Table I. Maternal demographics and antenatal investigations ( $n=29)$.

\begin{tabular}{|c|c|}
\hline Variable & No. (\%) \\
\hline Maternal age* (yrs) & $30(23-42)$ \\
\hline Gestational age at diagnosis ${ }^{\dagger}$ (wks) & $27(12-37)$ \\
\hline Multigravidity & $17(59)$ \\
\hline Singleton pregnancy & $28(97)$ \\
\hline Thalassaemia screening & $29(100)$ \\
\hline Chromosomal analysis & $19(66)$ \\
\hline Intrauterine infection screening & $17(59)$ \\
\hline
\end{tabular}

${ }^{*}$ Data is expressed as mean (range). ${ }^{\dagger}$ Data is presented as median (range).

Table II. Neonatal demographics $(n=19)$.

\begin{tabular}{lc}
\hline Variable & No. (\%) \\
\hline Gestational age at birth* (wks) & $33(27-37)$ \\
Mode of delivery & \\
$\quad$ Lower-segment caesarean section & $12(63)$ \\
$\quad$ Normal vaginal delivery & $7(37)$ \\
Median birth weight* ${ }^{\text {(g) }}$ & $2,480(1,230-3,900)$ \\
Sex & \\
Male & $13(68)$ \\
Female & $6(32)$ \\
Apgar score* & \\
At 1 min & $3(0-9)$ \\
At 5 mins & $7(1-9)$ \\
Resuscitation needed & \\
Yes & $17(89)$ \\
No & $2(11)$ \\
\hline
\end{tabular}

${ }^{*}$ Data is expressed as median (range).

diagnosed with NIHF was a twin pregnancy. The median gestational age at diagnosis of NIHF was 27 (range 12-37) weeks.

The significant neonatal demographics are indicated in Table II. The median gestational age at birth was 33 (range 27-37) weeks and the median birth weight was 2,480 (range 1,230-3,900) g. Ethnically, Chinese comprised the majority $(77 \%)$ although other ethnic groups were also represented (2 [7\%] Malay, 1 [3\%] Filipino, 1 [3\%] Thai, 1 [3\%] Cambodian, 1 [3\%] Caucasian). There were 19 live births, with a male to female ratio of almost 2.2:1. The median Apgar score of the live births at 1 min was 3 (range 0-9), and at 5 mins was 7 (range 1-9). Only two live births did not require any active resuscitation. All the neonates of the remaining 17 live births were intubated; 11 required thoracocentesis or abdominal paracentesis at birth, and 7 were given endotracheal or intravenous adrenaline. Despite the efforts made, six neonates died in the delivery room. Of the 11 neonates admitted to the neonatal intensive care unit, 7 required high frequency oscillation ventilation while 4 were on conventional ventilation. A total of six neonates were on inotropic support.

Of the 29 cases with a diagnosis of NIHF, a plausible cause was observed in 20 (69\%) women. No cause could be determined in the remaining $9(31 \%)$ cases. The degree of evaluation received by the cases diagnosed with NIHF varied. Only 19 out of 29 (66\%) cases underwent karyotype
Table III. Aetiology of nonimmune hydrops fetalis $(\mathbf{n}=29)$.

\begin{tabular}{ll}
\hline Variable & No. (\%) \\
\hline Cardiovascular & $5(17)$ \\
Complex cyanotic heart disease & $2(7)$ \\
Complete heart block & $1(3)$ \\
Ebstein's anomaly & $1(3)$ \\
Supraventricular tachycardia & $1(3)$ \\
Haematological & $4(14)$ \\
Bart's hydrops & $2(7)$ \\
Fetomaternal haemorrhage & $1(3)$ \\
Twin-to-twin transfusion syndrome & $1(3)$ \\
Congenital tumour & $4(14)$ \\
Giant haemangioma & $3(10)$ \\
Sacrococcygeal teratoma & $1(3)$ \\
Cystic hygroma & $3(10)$ \\
Genetic/metabolic & $4(14)$ \\
Chromosome 7q34 deletion & $1(3)$ \\
Congenital disorder of glycosylation type $1 \mathrm{a}$ & $1(3)$ \\
Kabuki syndrome & $1(3)$ \\
Turner's syndrome with cystic hygroma & $1(3)$ \\
Idiopathic & $9(31)$ \\
\hline
\end{tabular}

evaluation. A total of 17 out of 29 (59\%) women were screened for intrauterine infections (IUI). However, these IUI investigations were often incomplete - 41\% (12/29) of women were screened for Toxoplasma, cytomegalovirus and parvovirus infection; 58\% (11/19) for rubella and 26\% (5/19) for herpes simplex virus. All mothers had a full blood count done antenatally as a screening test for thalassaemia. If the mean corpuscular volume in any mother was $<80 \mathrm{fL}$, haemoglobin electrophoresis was offered. Of the 9 mothers who underwent haemoglobin electrophoresis, 5 were found to have thalassaemia trait (excluding one who was already known to have $\mathrm{HbH}$ disease). Cardiac evaluations in the form of either postnatal echocardiography or postmortem examination were performed for $72 \%(21 / 29)$ of the neonates.

A complete workup was done in 4 of the 9 cases without any known cause for NIHF. Of these 9 cases, 7 (78\%) had normal chromosomes, 6 (67\%) were negative for IUI; all were screened for thalassaemia and unremarkable cardiac findings were found in $6(67 \%)$ mothers. For the remaining 20 cases with known aetiology, the common diagnoses were: (a) cardiac anomalies; (b) haematological problems, including homozygous alpha thalassaemia (Bart's hydrops), twin-to-twin transfusion syndrome (TTTS) and fetomaternal haemorrhage; (c) congenital tumours, including haemangioma and teratoma; and (d) cystic hygromas. Two cases had chromosomal disorder, one had a congenital disorder of glycosylation type 1a and one was diagnosed with Kabuki syndrome (Table III).

There were a total of 19 live births, 1 intrauterine death, and 9 medical terminations of pregnancy following the diagnosis of hydrops fetalis. Of the 19 live births, 8 neonates survived and 11 died during the neonatal period (Fig. 1). Four of the survivors had no identified aetiology of NIHF. The neonatal mortality rate was approximately $58 \%$. The causes and outcomes of NIHF cases in our series are illustrated in Fig. 2. 


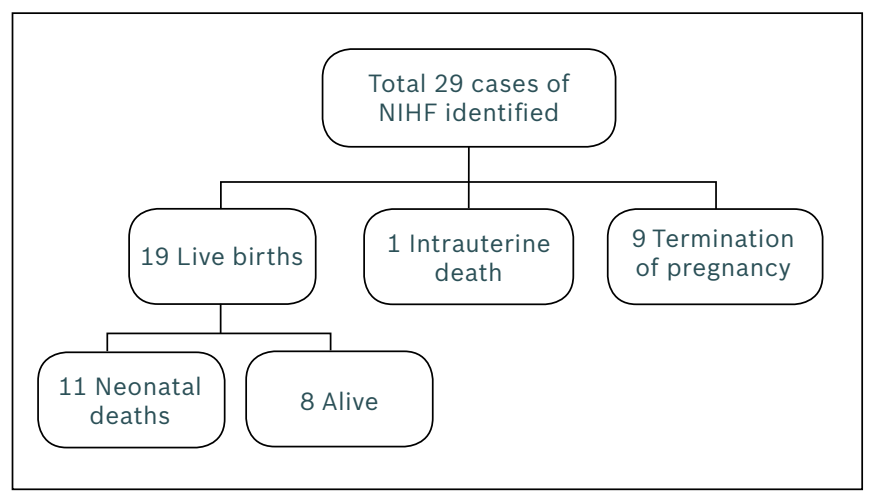

Fig. 1 Flow chart shows perinatal and neonatal outcomes of the cases in the study group.

\section{DISCUSSION}

The number of conditions associated with NIHF is extensive. In a recent systematic review of NIHF, Bellini et al found that cardiovascular causes were the most common (21.7\%) aetiological factor. ${ }^{(1)}$ According to these authors, chromosomal, syndromic and metabolic disorders collectively made up 18.9\% of NIHF cases, while haematological and TTTS-placental conditions comprised $16 \%$ of cases. ${ }^{(1)}$ This is comparable with the results in our series, in which we found a majority of NIHF cases to be associated with cardiac anomalies $(17 \%)$, haematological causes $(14 \%)$ and genetic/metabolic disorders (14\%). In our study, other important factors were congenital tumours (14\%) and cystic hygromas (10\%).

In our series, 2 of the $29(7 \%)$ NIHF cases were caused by homozygous alpha-thalassaemia. Interestingly, this number is relatively low compared to that of other Asian countries. For instance, in a ten-year review of hydrops fetalis at a hospital in Thailand, the most common known cause was Bart's hydrops fetalis $(36.6 \%)$. $^{(3)}$ Similarly, a four-year series from a hospital in Taiwan also found the most common cause of NIHF to be Bart's hydrops fetalis (31\%). ${ }^{(4)}$ As the gene prevalence of thalassaemia in our local population is relatively high at approximately $3 \%$, nationwide antenatal screening for thalassaemia is implemented. ${ }^{(5)}$ Since the introduction of routine thalassaemia screening, the number of beta-thalassaemia major and homozygous alpha-thalassaemia births in Singapore has decreased dramatically. ${ }^{(6)}$ This probably contributed to the lower numbers of Bart's hydrops fetalis found in our study compared to those from neighbouring countries.

Knowledge of the aetiology of a condition implies a better understanding of the underlying pathophysiology, which enables improved prevention and management strategies to be instituted. However, there remains a large number of NIHF cases for which the cause remains unknown. In our study, almost one-third of the NIHF cases had no identified aetiology. The review by Bellini et al found $17.8 \%$ of all cases reviewed to be idiopathic, ${ }^{(1)}$ while another recent review by Abrams et al, which consisted of 598 NIHF cases from a large national database, found that the aetiology in $26.3 \%$ of cases were unidentified.(7) In the past, no cause was found in

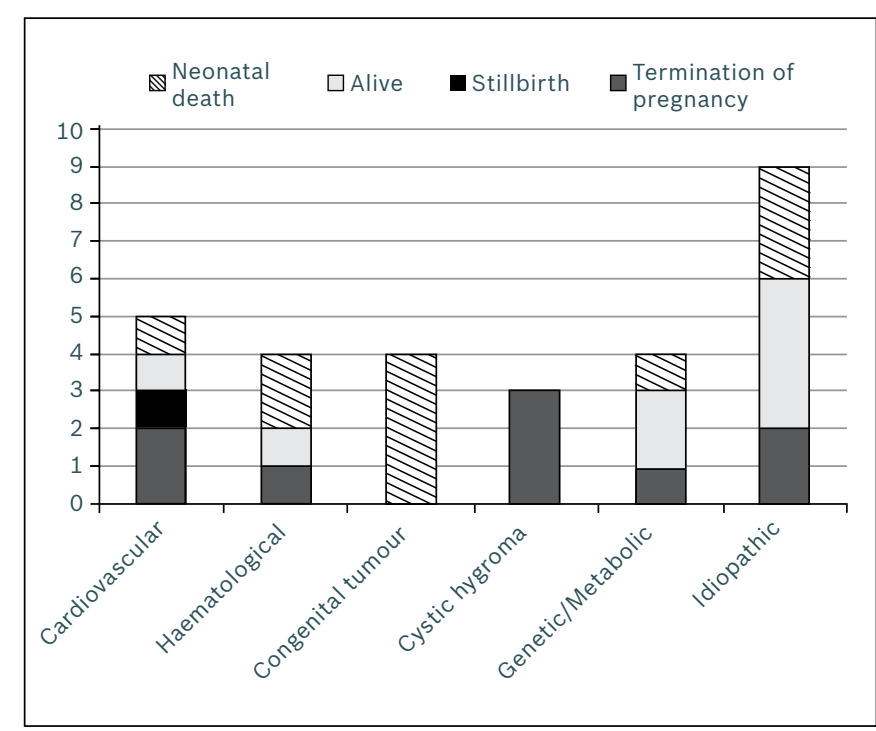

Fig. 2 Graph illustrates the outcome of cases in each aetiological group $(n=29)$.

approximately $50 \%$ of NIHF cases. ${ }^{(2)}$ However, with complete prenatal and postnatal evaluations, it is now suggested that a precise diagnosis can be arrived at in up to $85 \%$ of NIHF diagnoses. ${ }^{(2)}$ Therefore, it is important to thoroughly investigate all NIHF cases so that common and known causes are not missed.

In our study, no case of NIHF was caused by congenital infection. However, only 17 of the 29 (59\%) NIHF cases were investigated for IUI, and of the 9 cases in which the aetiology of NIHF was not identified, only 4 had a complete evaluation. Even in these four cases, the IUI workup was inconsistent. Thus, there is a need for a systematic and stepwise approach in NIHF investigations and diagnosis to ensure standardised evaluation of all cases.

As diagnostic and therapeutic strategies improve, it is hoped that the prognosis of NIHF will also improve. In our study, the neonatal mortality rate was almost $58 \%$. The mortality rates in other centres range from $40 \%$ to $95 \%{ }^{(8-10)}$ In the review by Abrams et al, the mortality rate was the highest among neonates with congenital anomalies (57.7\%) and lowest among neonates with congenital chylothorax (5.9\%). ${ }^{(7)}$ According to Stephenson et al, the mortality rate was lower if the hydrops was due to fetal supraventricular tachycardia (SVT) that responded to maternal treatment. ${ }^{(8)}$ The only neonate with hydrops secondary to fetal SVT in our study survived.

Better techniques for antenatal screening have made the earlier detection of NIHF possible. In our series, the median gestational age at diagnosis of NIHF was 27 weeks. Antenatal identification of the aetiology of NIHF would allow for appropriate counselling of parents and help clinicians provide parents with early options such as medical termination of pregnancy, planned delivery or active antenatal treatment.

The median gestational age at birth was 33 weeks in our study. However, one should bear in mind that the decision on the timing of delivery should be jointly made by the 
obstetrician and neonatologist, as the risks of preterm delivery and hydrops should not be taken lightly. Furthermore, there have also been reported cases of spontaneous intrauterine resolution of fetal ascites and effusions. ${ }^{(11)}$ We encountered two such cases of spontaneous resolution in our study; these neonates required no active resuscitation at birth.

As this was a retrospective study, it was limited by incomplete data. As mentioned earlier, investigations were inconsistently performed in the cases diagnosed with NIHF in our cohort. We were unable to obtain information on the severity of prenatal presentation or intervention. There was also no information on the long-term outcome of infants who survived. Apart from this, the number of cases diagnosed with NIHF at our hospital over the six-year study period was low. Larger prospective studies that assess the long-term neurodevelopmental outcome of NIHF are therefore warranted. Systematic guidelines for the investigation of NIHF cases should also be put in place to ensure a comprehensive search of its aetiology.

In our series, almost one-third of cases antenatally diagnosed with NIHF had no identified aetiology, and the neonatal mortality rate was found to be approximately $58 \%$. Our findings highlight the importance of thorough investigation of all cases of NIHF to identify its causes, so that appropriate counselling and better management can be ensured for affected parents, both antenatally and postnatally.

\section{ACKNOWLEDGEMENT}

The authors would like to thank the Department of Neonatology, KK Women's and Children's Hospital, Singapore, for providing guidance and assistance during the course of the project. There was no conflict of interest during the study.

\section{REFERENCES}

1. Bellini C, Hennekam RC, Fulcheri E, et al. Etiology of nonimmune hydrops fetalis: a systematic review. Am J Med Genet A 2009; 149A:844-51.

2. Santolaya J, Alley D, Jaffe R, Warsof S. Antenatal classification of hydrops fetalis. Obstet Gynecol 1992; 79:256-9.

3. Ratanasiri T, Komwilaisak R, Sittivech A, Kleebkeaw P, Seejorn K. Incidence, causes and pregnancy outcomes of hydrops fetalis at Srinagarind Hospital, 1996-2005: a 10-year review. J Med Assoc Thai 2009; 92:594-9.

4. Yang YH, Teng RJ, Tang JR, et al. Etiology and outcome of hydrops fetalis. J Formos Med Assoc 1998; 97:16-20.

5. Yeo GS, Tan KH, Liu TC. The role of discriminant functions in screening for beta-thalassaemia traits during pregnancy. Singapore Med J 1995; 36:615-8.

6. Yeo GS. Inaugural College of Obstetricians \& Gynaecologists Lecture: recent developments in obstetric care and maternal fetal medicine in Singapore. Ann Acad Med Singapore 2004; 33:738-42.

7. Abrams ME, Meredith KS, Kinnard P, Clark RH. Hydrops fetalis: a retrospective review of cases reported to a large national database and identification of risk factors associated with death. Pediatrics 2007; 120:84-9.

8. Stephenson T, Zuccollo J, Mohajer M. Diagnosis and management of non-immune hydrops in the newborn. Arch Dis Child Fetal Neonatal Ed 1994; 70:F151-4.

9. Trainor B, Tubman R. The emerging pattern of hydrops fetalis--incidence, aetiology and management. Ulster Med J 2006; 75:185-6.

10. Ismail KM, Martin WL, Ghosh S, Whittle MJ, Kilby MD. Etiology and outcome of hydrops fetalis. J Matern Fetal Med 2001; 10:175-81.

11. Platt LD, Collea JV, Joseph DM. Transitory fetal ascites: an ultrasound diagnosis. Am J Obstet Gynecol 1978; 132:906-8. 